www.nature.com/pj

\title{
Dissipative particle dynamics simulation on the association properties of fluorocarbon-modified polyacrylamide copolymers
}

\author{
Tao Ni, Guang-Su Huang, Pin Gao, Yun-tao Xu and Ming-Zhu Yang
}

The hydrophobic association properties of a modified polyacrylamide (HMPAM) solution are attributed to the composition of the solution and environmental factors. In this study, the hydrophobic association of fluorocarbon-modified polyacrylamide aqueous solutions has been investigated on a mesoscopic level by considering the variation of root mean square (RMS) end-to-end distances using dissipative particle dynamics (DPD) simulations. The hydrophobic association depends on the hydrophobic behavior of the groups incorporated, the environmental temperature, the addition of salts and the shear. The embedded fluorocarbon units in the polyacrylamide chains are trifluoroethyl methacrylate, hexafluorobuyl methacrylate and dodecafluoroheptyl methacrylate, respectively, and the corresponding copolymers are expressed as P(AM-AANa-3F), $P(A M-A A N a-6 F)$ and $P(A M-A A N a-12 F)$. The modeling results show that $P(A M-A A N a-12 F)$ has the strongest hydrophobic interactions among the three copolymers. The hydrophobic association of $\mathrm{P}(\mathrm{AM}-\mathrm{AANa}-12 \mathrm{~F})$ solutions in pure water is lower than that of corresponding salt-water solutions and shows stronger positive salinity sensitivity. P(AM-AANa-12F) can maintain its hydrophobic association in aqueous solution at high temperature, and its three-dimensional network can be reformed after shear. These conclusions are consistent with the experimental and theoretical results.

Polymer Journal (2011) 43, 635-641; doi:10.1038/pj.2011.24; published online 25 May 2011

Keywords: dissipative particle dynamics; fluorocarbon-modified polyacrylamide; hydrophobic association

\section{INTRODUCTION}

Hydrophobically modified water-soluble polymers have been extensively studied over the past two decades in both academic and industrial laboratories. In particular, hydrophobically modified polyacrylamide (HMPAM), obtained by incorporating a relatively small amount of hydrophobic monomer into the polyacrylamide chain, has received increasing attention because of its unique association characteristics and wide applications in the fields of enhanced oil recovery, drilling fluids and drug release. ${ }^{1-5}$ As a result, great efforts have been made in the preparation and characterization of HMPAM. There are two synthetic strategies for incorporating hydrophobic moieties into water-soluble polymer chains: post-polymerization functionalization and direct copolymerization of hydrophobic and water-soluble monomers. The structure of HMPAM prepared by post-modification ${ }^{6-7}$ cannot be well controlled and possesses a statistical distribution of the hydrophobic groups along the chain and homogeneous composition. Micellar ${ }^{8-10}$ and inverse-phase ${ }^{11}$ copolymerization can obtain inhomogeneous and amphiphilic block copolymers. With respect to characterization, ${ }^{1} \mathrm{H}$ and ${ }^{13} \mathrm{C}^{12}$ are commonly used to characterize composition and chain construction in detail; static light scattering ${ }^{13}$ and automatic mixing technologies ${ }^{14}$ can be used to determine some structural factors of polymer samples. Moreover, fluorescence ${ }^{15}$ analysis can be used to confirm whether a hydrophobic block is sustainable.

Although much progress has been made on the preparation and characterization of HMPAM, this research is still in its infancy and the corresponding knowledge produced is scarce. The influence of chain architecture and composition on the morphology of HMPAM is very important and must be explored within a wide parameter space, requiring a tremendous amount of experimental effort. However, changing experimental parameters in a systematic manner may also be hindered by technical difficulties. Furthermore, it is difficult to detect the nature of hydrophobic association or visualize the evolution of the association directly by experiment. However, molecular simulation is a powerful tool that can complement experimental work not only because it operates within a wider parameter space but also because it is less costly yet more effective. Molecular simulations before experimental investigations can provide a preview of the 
answers to questions that can guide future experimental research and lead to valuable molecular-level information. The simulations can provide useful information that may contribute to future rational design and the synthesis of novel anti-salt and anti-temperature polyacrylamide.

At present, hydrocarbon groups are introduced into polyacrylamide as hydrophobic groups, but fluorocarbon groups may be better in this respect, because they possess stronger hydrophobic properties ${ }^{16-17}$ and lower cohesion density and surface energy compared with hydrocarbon groups of the same carbon-chain length. Thus, this article presents a study of fluorocarbon-modified polyacrylamide by molecular simulation.

\section{EXPERIMENTAL PROCEDURE}

\section{Simulation theory and method}

Molecular simulation is a new computational chemistry developed by the combination of computer science and theoretical chemistry. According to the dimension of the modeling system, molecular simulations include molecularscale simulation and mesoscale simulation. The simulation methods used to study molecular-scale interactions include quantum mechanics, molecular mechanics, molecular dynamics (MD) and Monte Carlo methods. Among these methods, MD simulation is the most widely applied method in the fields of chemistry, biology and materials science. The programs used for MD simulation are numerous and include softwares such as NAMD, AMBER, CHARM, TINKER, LAMPS and GROMACS. Among these, AMBER, CHARM and GROMACS are focused on modeling biological systems, and their force fields are mainly dictated by a limited number of atoms. Thus, they are greatly limited in application to non-biological systems. The other programs used for MD simulation can be used in both biological and non-biological systems, but the large size and slow dynamics of mesoscale structures make them prohibitively expensive; even complex fluids cannot be modeled over certain length and timescales using these programs. The molecular simulation methods used to model mesoscopic scale systems are currently at the forefront of computational chemistry. The comparatively mature simulation methods are mainly mesoscopic dynamics (meso dynamics) and dissipation particle dynamics (DPD). It is a coarse-grained simulation technique originally developed by Hoogerbrugger and Koelman. ${ }^{18-19}$ This technique allows for the simulation of hydrodynamic behavior in much larger and more complex systems than the classic molecular dynamics and Monte Carlo simulations, which can be captured up to the microsecond range. Recently, this method has been successfully used to study the microstructure and properties of polymers in their bulk state and in solution. ${ }^{20-23}$ In solution, the systems treated by DPD modeling are typically mixtures of several components that exhibit self-organization on the nanometer scale, such as dilute or semi-dilute polymer solutions, suspensions of colloidal particles, vesicles and cells and microemulsions. Thus, DPD is a very promising simulation tool for modeling polymer solutions that can focus directly on the length and timescales that are difficult to probe experimentally and cannot be easily addressed by conventional simulation tools. With the aim of achieving a better understanding of hydrophobic association, the DPD technique has been used in this work to study the self-assembly of acrylamide-fluorocarbonacrylate copolymers.

In the DPD method, a series of soft particles is assumed to interact with each other, and each particle represents a small volume of fluid containing a few atoms. In addition, the forces acting on a particle include conservative forces, dissipative forces and random forces, each of which is pairwise additive:

$$
f_{i}=\sum_{j \neq i}\left(F_{i j}^{\mathrm{C}}+F_{i j}^{\mathrm{D}}+F_{i j}^{\mathrm{R}}\right)
$$

where the sum runs over all beads within the cutoff radius (rc); rc is set to unity (DPD unit). The conservative force is a soft-repulsion central force and is given by

$$
F_{i j}^{C}= \begin{cases}a_{i j}\left(1-\left|r_{i j}\right|\right) \hat{r}_{i j} & \left(\left|r_{i j}\right|<1\right) \\ 0 & \left(\left|r_{i j}\right| \geq 1\right)\end{cases}
$$

where $a_{i j}$ is the maximum repulsion between particles $i$ and $j, \vec{r}_{i}$ is the position vector of particle i, $\vec{r}_{i j}=\vec{r}_{i}-\vec{r}_{j}$, and $\hat{r}_{i j}=\vec{r}_{i j} /\left|\vec{r}_{i j}\right|$. The dissipative force is given by

$$
F_{i j}^{D}=-\gamma \omega^{D}\left(r_{i j}\right)\left(\hat{r}_{i j} \cdot \vec{v}_{i j}\right) \hat{r}_{i j}
$$

and the random force is given by

$$
F_{i j}^{R}=\sigma \omega^{R}\left(r_{i j}\right) \xi_{i j} \hat{r}_{i j}
$$

where $\vec{v}_{i}$ is velocity of particle i, $\vec{v}_{i j}=\vec{v}_{i}-\vec{v}_{j}, \omega\left(r_{i j}\right)$ is the weight function, $\sigma$ is the noise amplitude and $\xi_{i j}$ is a randomly fluctuating variable with Gaussian statistics.

The dissipative and random force can been considered thermal baths, which function as a thermostat and conserve angular momentum.

To determine the conservative force $\left(F^{c}\right)$, the repulsion parameter $a_{i j}$ has to be known. Groot et al. ${ }^{20}$ proposed the relationship between $a_{i j}$ and the Flory-Huggins parameter $x$ as follows:

$$
a_{i j}=\left\{\begin{array}{lll}
a_{i i}+3.27 x_{i j} & \rho=3 & \text { (DPD unit) } \\
a_{i i}+1.45 x_{i j} & \rho=5 & \text { (DPD unit) }
\end{array}\right.
$$

where $\rho$ is the density, $a_{i i}$ is the repulsion parameter between particles of the same type and its value is derived from the compressibility of the pure component (Supplementary Information).

$$
a_{i i}=75 \mathrm{kBT} / \rho
$$

In the DPD method, the timer unit $\tau$ is given by

$$
\tau=r_{c} \sqrt{m / k_{B} T}
$$

where $m$ is the unit of mass and $k_{\mathrm{B}} T$ is the thermal energy as well as the unit of energy. Therefore, the timescale can be established by

$$
\bar{t}=\frac{t}{\sqrt{m r_{\mathrm{c}}^{2} / k_{B} T}}
$$

where $\bar{t}$ is time in DPD units. Alternatively, the timescale could also be determined by comparing the diffusion of the bead to the known self-diffusion coefficient of the molecule that it represents.

To characterize the chemical properties, input parameters, including the number of beads in each chain and interactions between beads, must be obtained. There are several methods suggested in the literature for evaluating the interaction parameters in DPD simulations. The quantitative structureproperty relationship (QSPR) method is based on the solubility parameter, which is not appropriate for the present system because of the presence of partially charged molecules. Although blends can also be used to calculate the interaction parameter of a charged polymer chain solution by all-atom simulation, it cannot reflect changes in the system temperature. Molecular mechanics and dynamics methods are usually more accurate, allowing for consideration of the temperature, salt strength and charged polymer chain. Therefore, the interaction parameters for DPD simulation have been calculated by both MM and MD. First, the energy of mixing is obtained using:

$$
\Delta \tilde{E}_{\text {mix }}=\Phi_{i}\left(\frac{E_{\mathrm{coh}}}{V}\right)_{i}+\Phi_{j}\left(\frac{E_{\mathrm{coh}}}{V}\right)_{j}-\left(\frac{E_{\mathrm{coh}}}{V}\right)_{\text {mix }}
$$

where $\varphi_{i}$ and $\varphi_{j}$ represent the volume fraction of component $i$ and $j$, respectively, and $\left(E_{\mathrm{coh}} / V\right)$ is the cohesive energy density of each component. All of these parameters can be calculated by analyzing the MD simulation results.

Further, the Flory-Huggins parameter is calculated by

$$
\chi=\left(\Delta \tilde{E}_{\text {mix }} / R T\right) V_{\text {mon }}
$$

where $V_{\text {mon }}$ is the molar volume of one monomer, calculated using the arithmetic average molar volume of the pair, $\mathrm{R}$ is the gas constant and $\mathrm{T}$ is the absolute temperature.

Finally, the Flory-Huggins parameter is put into one equation of the formula (5), and the interaction in the DPD simulation is obtained. 
a<smiles>C=C(C)C(=O)OCC(F)(F)F</smiles>

b

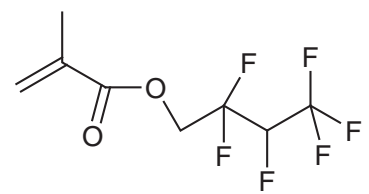

C<smiles>C=C(C)C(=O)OCC(F)(F)C(F)C(F)(F)C(F)(F)C(F)(F)C(F)(F)F</smiles>

Figure 1 Structures of fluorocarbon acrylates: (a) trifluoroethyl methacrylate; (b) hexafluorobutyl methacrylate; and (c) dodecafluoroheptyl methacrylate.

The number of beads in each mesoscopic molecule is determined by the polymerization $\left(D_{\mathrm{n}}\right)$ and the characterization ratio $\left(C_{\mathrm{n}}\right)$ of the polymer, and the expression for DPD chain length $\left(\mathrm{N}_{\mathrm{DPD}}\right)$ is as follows:

$$
N_{\text {DPD }}=\frac{D_{n}}{C_{n}}
$$

The $V_{\text {mon }}$ and $C_{\mathrm{n}}$ cannot be calculated by MD, but they can be obtained by the quantitative structure-property relationship method, such as that available in the Synthia module.

In this study, fluorocarbon-modified polyacrylamide was composed of partially hydrolyzing polyacrylamide (HPAM) and polyfluorine-carbon acrylate. The fluorocarbon acrylates involved in this study were mainly trifluoroethyl methacrylate (3F), hexafluorobuyl methacrylate $(6 \mathrm{~F})$ and dodecafluoroheptyl methacrylate (12F), as shown in Figure 1.

In DPD simulations, HPAM and polyfluorine-carbon acrylate are treated as hydrophilic (A) and hydrophobic particles (B), respectively; 'A' particles have partial charge. 'A' copolymer solution contains water and salt solutions; the salt solution contains a monovalent salt, namely, sodium chloride. Both water solution and salt solution are denoted as W. Detailed information regarding these solutions is given in Figure 2.

To calculate the average pair-interaction energy $E_{i j}$, the pure HPAM system, the pure polyfluorine-carbon acrylate system and a mixed system of HPAM, polyfluorine-carbon acrylate and solvent are constructed by using Amorphous Cell under the COMPASS force field, which is the most up-to-date and accurate force field for the calculation of molecular interactions in aqueous systems. MM and MD simulations of the obtained systems were performed. These generate values for the cohesive energy density and volume fraction of each system. Further, the molar volume and monomer characteristic ratio of the species were calculated by the Synthia module, and the interaction parameters $a_{i j}$ of each pair of these (that is, A-B, A-W and B-W) were calculated using Equations (5), (9) and (10). The calculated $a_{i j}$ parameters are dependent on the temperature and solvent properties, and the involved $a_{i j}$ parameters are given in Tables 1-3, respectively (Supplementary Information). To avoid intramolecular aggregation, the structure of the copolymers was designed as A500B6A300. The dissipative parameter $\gamma$ was set to a value of $4.5 k_{\mathrm{B}} T$. In all simulations, reduced units were used. The only length scale in the system was the cutoff radius $\mathrm{rc}$, which was the length unit in the simulation. The particle density of all of the systems was set to $\rho=3$. The step size for integration of the Newton equation was $\Delta t=0.04$. The box size for the simulation was set to $(15 \times 15 \times 15)$ in units. All DPD particles belonging to the same molecule were connected by a loosely bound spring with a spring force constant $\mathrm{K}=4$. This spring constant controls the stiffness of the molecule but is not very sensitive to the simulation results. For each system, 20000 steps per simulation were performed. All calculations were performed by Materials Studio (Accelrys Company, San Diego, CA, USA).

\section{RESULTS AND DISCUSSION}

\section{Effects of hydrophobic group on association properties} of fluorocarbon-modified polyacrylamide

The root mean square (RMS) end-to-end distance $\left(h^{2}\right)^{1 / 2}$ is a parameter of polymer chain dimension, which describes the degree of curliness of a polymer chain. In this article, $\left(h^{2}\right)^{1 / 2}$ is regarded as a parameter of the conformation and dimension for a fluorine carbonmodified single polyacrylamide chain in solution. In dilute solution, each of the polyacrylamide chains modified by fluorine carbon are
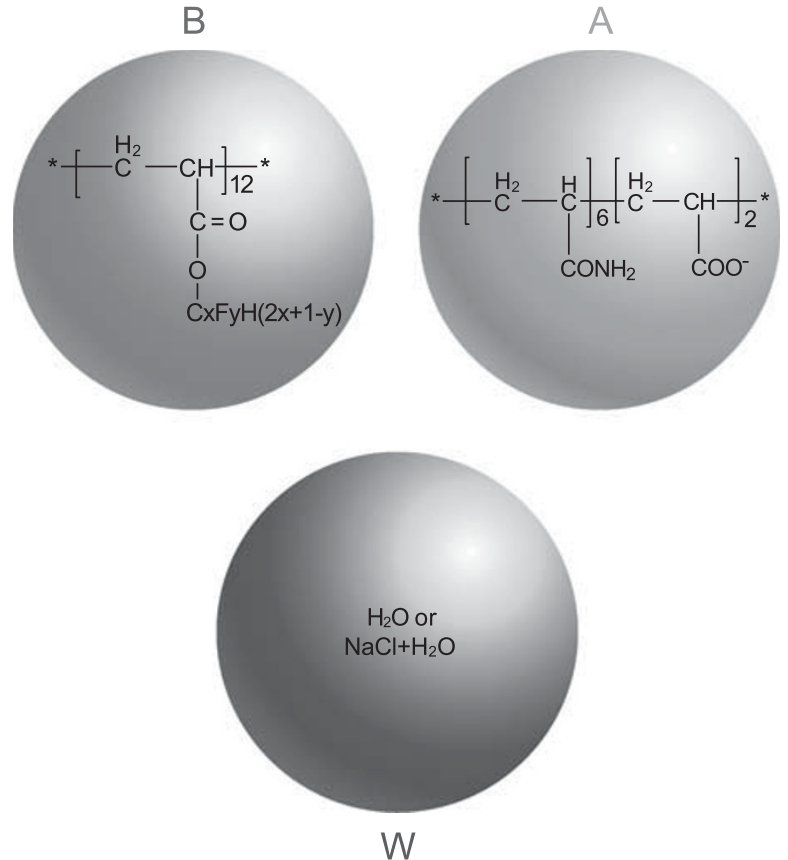

Figure 2 Schematic representation of acrylamide, fluorocarbon acrylate and water beads in the dissipative particle dynamics (DPD) simulation.

treated as isolated entities, and the intermolecular interaction can be ignored, which results in a stretched conformation and a greater $\left(h^{2}\right)^{1 / 2}$ value for the macromolecular chain. When the concentration increases to the critical association concentration $\left(\mathrm{C}^{\star}\right)$, intermolecular aggregations of the fluorocarbon-modified polyacrylamide are formed by hydrophobic groups, and the space of the hydrophobic domain is relatively concentrated, which does not allow macromolecule chains to sufficiently stretch so that the value $\left(h^{2}\right)^{1 / 2}$ of the chains reduces in solution. Above the $C^{\star}$, the association network volume and association network number increase with the extent of intermolecular association, resulting in a greater degree of curliness in the macromolecular chain. On the basis of this analysis, the greater the association, the smaller the value of $\left(h^{2}\right)^{1 / 2}$ for the macromolecular chain.

As shown in Figure 3, an increase in RMS end-to-end distance occurs with increasing concentration and then declines for three copolymers: $\mathrm{P}(\mathrm{AM}-\mathrm{AANa}-3 \mathrm{~F}), \mathrm{P}(\mathrm{AM}-\mathrm{AANa}-6 \mathrm{~F})$ and $\mathrm{P}(\mathrm{AM}-\mathrm{AANa}-$ $12 \mathrm{~F})$. The increase results from the stretched conformation of the macromolecular chain in the dilute solution. With an increase in concentration, the number of ionic groups increases, the solvent molecules form hydrolysis layers and the structure also becomes stronger. On the other hand, with the increase in concentration, the density of the hydrophobic group increases, the hydrogen bonds of water molecules are interrupted and the ordering of water molecules increases. Because of these effects, the solvent prevents macromolecules from moving in the water solution, and the number of possible 
Table 1 Interaction parameters $a_{i j}$ between the different beads used in water solution at $298 \mathrm{~K}$ for the simulations

\begin{tabular}{lrrccc}
\hline & $W$ & $A$ & $B(3 F)$ & $B(6 F)$ & $B(12 F)$ \\
\hline W & 25.00 & 11.99 & 107.34 & 137.29 & 196.12 \\
A & 11.99 & 25.00 & 281.04 & 353.54 & 484.62 \\
B(3F) & 107.34 & 281.04 & 25.00 & $/$ & $/$ \\
B(6F) & 137.29 & 353.54 & $/$ & 25.00 & $/$ \\
B(12F) & 196.12 & 484.62 & $/$ & $/$ & 25.00 \\
\hline
\end{tabular}

Table 2 Interaction parameters $a_{i j}$ of beads for P(AM-AANa-12F) in salt solution at $298 \mathrm{~K}$ for the simulations

\begin{tabular}{lrrr}
\hline & $W$ & \multicolumn{1}{c}{$A$} & \multicolumn{1}{c}{$B(12 F)$} \\
\hline W & 25.00 & -23.53 & 249.09 \\
A & -23.53 & 25.00 & 484.62 \\
B(12F) & 249.09 & 484.62 & 25.00 \\
\hline
\end{tabular}

Table 3 Interaction parameters $a_{i j}$ of beads for P(AM-AANa-12F) in salt solution at $333 \mathrm{~K}$ for the simulations

\begin{tabular}{lrrr}
\hline & \multicolumn{1}{l}{$A$} & \multicolumn{1}{c}{$B(12 \mathrm{~F})$} \\
\hline W & 25.00 & -33.54 & 182.55 \\
A & -33.54 & 25.00 & 407.69 \\
B(12F) & 182.55 & 407.69 & 25.00 \\
\hline
\end{tabular}

configurations decreases, which increases the rigidity of the polymer chain, and the $\left(h^{2}\right)^{1 / 2}$ of the polymer is shown to increase in the aqueous solution. When the concentration of the solution reaches $\mathrm{C}^{*}$, intermolecular aggregations form and $\left(h^{2}\right)^{1 / 2}$ of the chains is reduced. The interaction between the macromolecules includes the repulsion interactions of the ionic groups and the association interactions of the hydrophobic groups. The former leads to a stretched conformation of the macromolecule chain, and the latter leads to a curled conformation of the macromolecule chain. Between these two factors, the latter is predominant at $C^{\star}$. When the concentration exceeds $C^{*}$ and as the space available for each polymer molecular decreases, the hydrophobic groups have a greater chance to aggregate and form a network structure, which leads to a further reduction in $\left(h^{2}\right)^{1 / 2}$ for the polymer chain.

From Figure 3, it can also be observed that the RMS end-to-end distance increases in the polymers in the following order: $\mathrm{P}(\mathrm{AM}$ AANa-3F), P(AM-AANa-6F) and P(AM-AANa-12F) at a certain concentration $\left(\leqslant 0.05 \mathrm{~mol} \mathrm{~mol}^{-1}\right)$. This trend can be explained from two perspectives. One is the hydrophobic effect of the fluorocarbon acrylate units in the polyacrylamide chains. The fluorocarbon acrylate fragments are weakly solvated in water because of the great differences in polarity, which leads to the ordering of water molecules and an increase in the rigidity of the polymer chain. The hydrophobicity of $12 \mathrm{~F}$ is the greatest among the above three fluorocarbon acrylates, as is its RMS end-to-end distance in the water solution. The spatial retarding effect is also the other reason for the trend observed. Beyond $0.05 \mathrm{~mol} \mathrm{~mol}^{-1}$, the order of increasing RMS end-to-end distance for the copolymers is contrary to that observed at lower concentration. The reason for this is that the association behaviors of the macro-

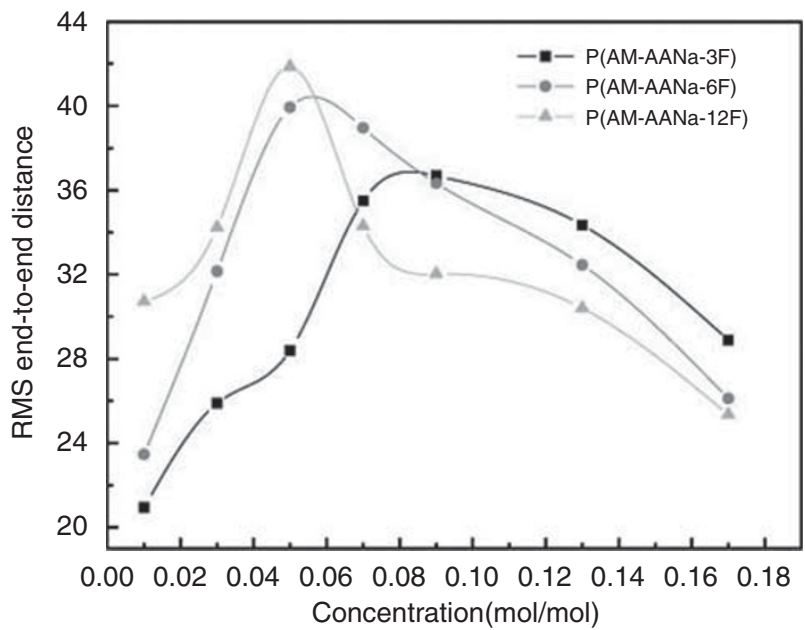

Figure 3 Root mean square end-to-end distance of P(AM-AANa-3F), P(AM$A A N a-6 F)$ and $P(A M-A A N a-12 F)$ solution at different concentrations.

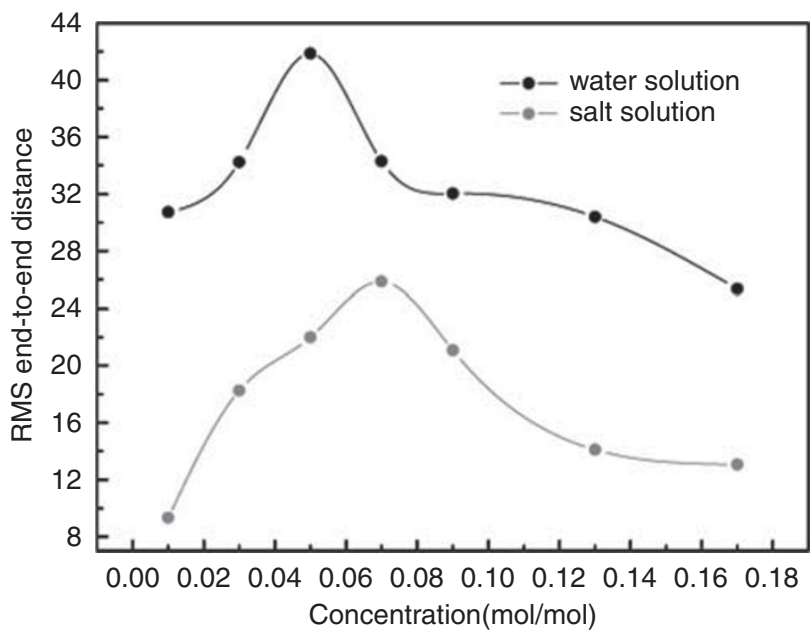

Figure 4 RMS end-to-end distance of $\mathrm{P}(\mathrm{AM}-\mathrm{AANa}-12 \mathrm{~F})$ salt solution at different concentrations.

molecules increase with the increase in hydrophobicity of fluorocarbon acrylate.

Effects of salt on the association properties of fluorocarbonmodified polyacrylamide

Because the hydrophobic association behavior of $\mathrm{P}(\mathrm{AM}-\mathrm{AANa}-12 \mathrm{~F})$ is more pronounced than that of the other two copolymers, only the effects of salt on the association properties of $\mathrm{P}(\mathrm{AM}-\mathrm{AANa}-12 \mathrm{~F})$ were studied in order to avoid repetitive discussions.

It can be observed in Figure 4 that the RMS end-to-end distance of $\mathrm{P}$ (AM-AANa-12F) first increases in the salt water solution, and then decreases with an increase in concentration. This trend is the same as that in the water solution, and the reason for this is also similar. However, the RMS end-to-end distance of the polymer chain in the salt-water solution is shorter than that in the water solution. Because of the screening of ions in the macromolecule by the addition of salt and the decrease in the repulsion interactions between these same ions, the macromolecule chain curls. Inorganic salts are generally considered to have a weak effect on the non-ionic fragments. However, 
a

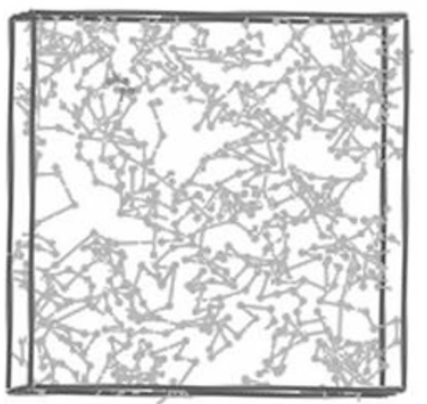

b

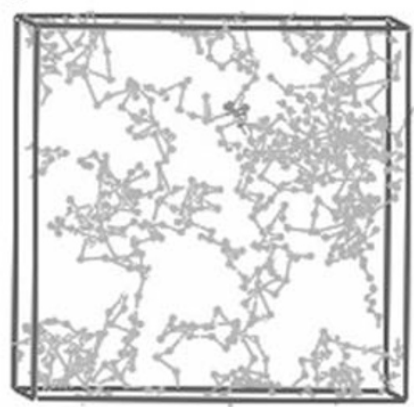

Figure 5 Mesomorphology of $\mathrm{P}$ (AM-AANa-12F) at 5\%: (a) water solution and (b) salt solution. a

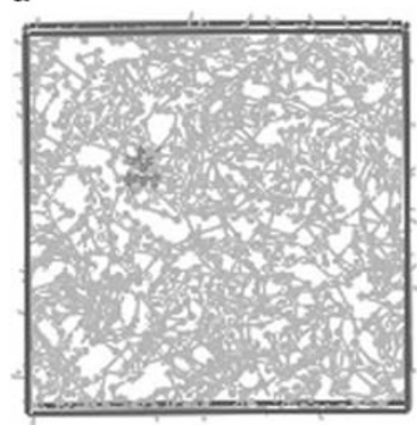

b

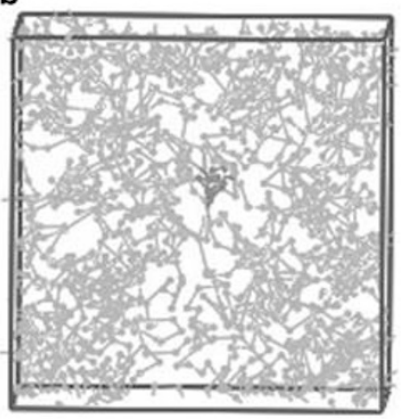

Figure 6 Mesomorphology of $\mathrm{P}$ (AM-AANa-12F) at 10\%: (a) water solution and (b) salt solution.

a large amount of inorganic salts would influence the solution properties by affecting the properties of water. When salts are introduced, dehydration of the group would be induced because the ions dissociated from the salts are strongly hydrated and break the hydrogen bonding between groups and water molecules, causing the salts to increase the repulsion parameter between that of the hydrophobic fragments and solvent molecules. Thus, the hydrophobic groups have a greater chance to associate; that is, they experience the so-called salting-out effect. The aforementioned reasons result in the curling of the polymer chains in the salt-water solution such that the RMS end-to-end distance is reduced. At low concentration, ion repulsion interactions determine the RMS end-to-end distance, whereas at high concentration, strong hydrophobic association is the predominant factor in determining the RMS end-to-end distance.

The mesomorphology of $\mathrm{P}$ (AM-AANa-12F) in water and salt solution is illustrated as follows in Figures 5 and 6 . In the figures, red beads represent hydrophobic groups, green beads represent hydrophilic groups and water molecules are removed. From the figures, it also can be seen that when the concentration is $5 \%$, a single molecule exists in a cell, and the molecule contracts in the salt-water solution with respect to its conformation in aqueous solution. In addition, when the concentration is $9 \%$, the macromolecule chain shows more pronounced association in the salt solution than in the water solution.

Effects of temperature on the association properties of fluorocarbon-modified polyacrylamide in salt solution

On the basis of the same reason that was discussed in the previous section, only the effects of temperature on the association properties of $\mathrm{P}(\mathrm{AM}-\mathrm{AANa}-12 \mathrm{~F})$ in salt solution were studied.

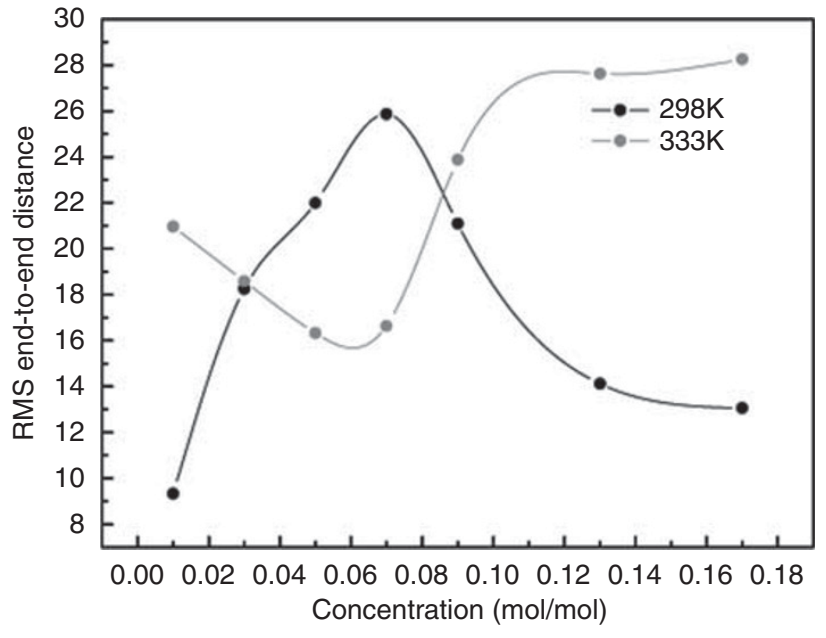

Figure 7 Temperature effects on the RMS end-to-end distance of P(AM-AANa-12F) in salt solution at different concentrations. a

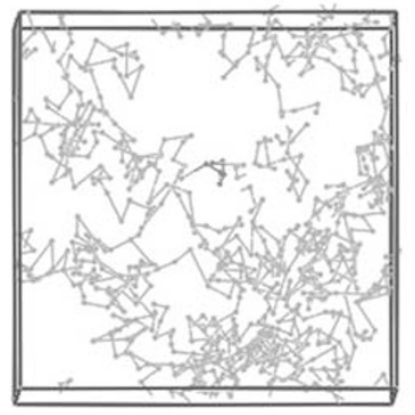

b

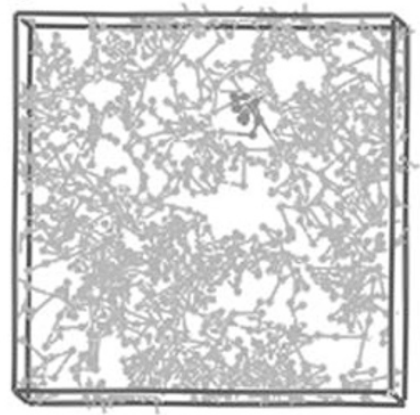

Figure 8 Mesomorphology of $P(A M-A A N a-12 F)$ in salt solution at $333 \mathrm{~K}$ : (a) 5 and (b) $9 \%$.

From Figure 7, we can conclude that the RMS end-to-end distance of $\mathrm{P}(\mathrm{AM}-\mathrm{AANa}-12 \mathrm{~F})$ in salt-water solution first decreases and then increases with an increase in the concentration at $333 \mathrm{~K}$. At higher temperature, the thermal motion of the hydrophobic groups of the macromolecule chain is enhanced, which weakens the ice structure of the water molecules around them and leads to the shrinking of the macromolecule thread. With the increase in concentration, the aggregation begins to form a physical network, and the network expands at higher temperature, as causes an increase in the RMS end-to-end distance. As the concentration continues to increase, the association behavior achieves relative balance, and the RMS end-to-end distance remains constant. In comparing Figure 6 with Figure 8, it can also be observed that, when the concentration is $5 \%$, the single molecule in the cell shrinks at $333 \mathrm{~K}$, compared with conformation at $298 \mathrm{~K}$. Moreover, when the concentration is $10 \%$, the association of the mesomolecules is stronger at higher temperatures.

\section{Effects of shear on association properties of fluorocarbon-modified} polyacrylamide

From Figure 9, it can be seen that the associating polymer solution is a uniform system of three-dimensional network structures before being sheared. When high-speed shear is applied to the solution, most of the associating parts of the polymer structure will be broken. When the shear force is removed, the ripped molecules associate to form a 
a

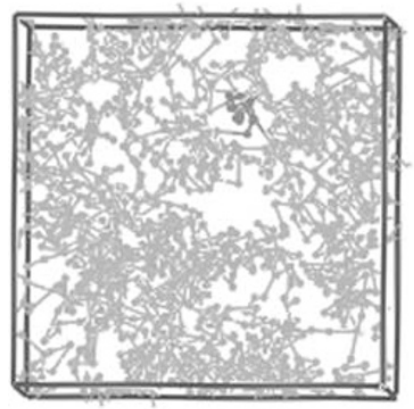

b

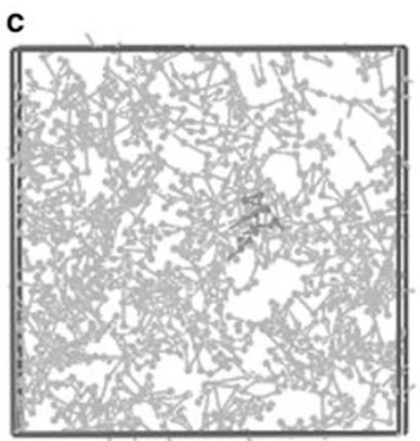

Figure 9 Mesomorphology of $\mathrm{P}$ (AM-AANa-12F) in salt solution:(a) before shear (b) during shear and (c) after shear.

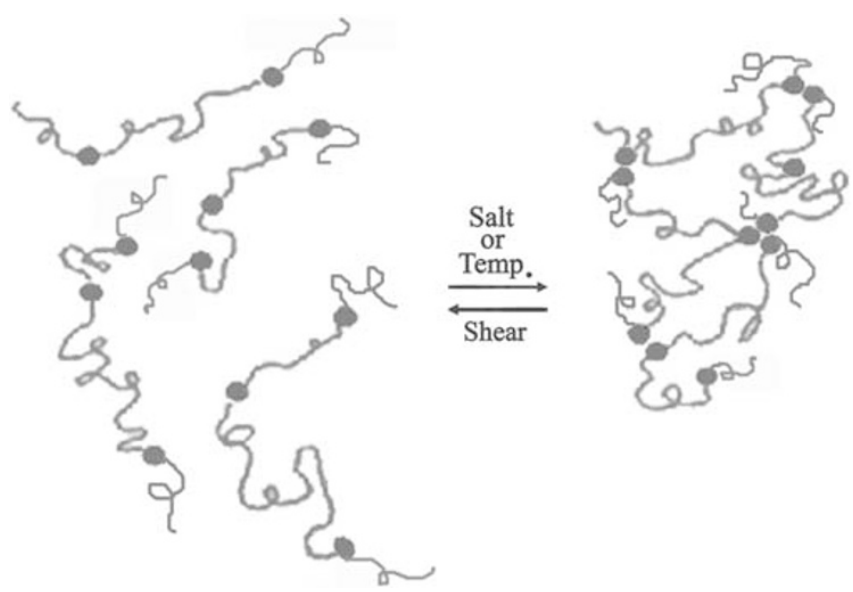

Figure 10 Association properties of fluorocarbon-modified polyacrylamide solution is affected by temperature, salt and shear.

continuous mesh structure. Because of its unique associative characteristics, the associating structure can reversibly change with the shear rate, contrary to the irreversibility of shearing a conventional high-viscosity polymer solution.

\section{Conclusion}

A hydrophobic fluorocarbonacrylate unit was incorporated into a polyacrylamide chain in a microblock manner, the aggregation of select copolymers was studied, and important findings were obtained. The hydrophobic association properties of acrylamide-fluorocarbonacrylate copolymers are affected by the type of hydrophobic group, salt, temperature and shear applied. The units with stronger hydrophobic properties within the chains produce the greatest association in the macromolecule in solution.
In addition, the copolymer solutions of fluorocarbon-modified polyacrylamide were shown to have strong positive salt and temperature sensitivity; that is, they thickened with increasing salt concentration and temperature. In addition, the three-dimensional network association structures can be restored after shear. Figure 10 clearly shows that environmental factors such as temperature, salt and shear have effects on the association of fluorocarbon-modified polyacrylamide. On the basis of the above results, it can be concluded that fluorine-carbon-modified polyacrylamide copolymers are materials that can potentially be used as enhanced oil recovery polymers for use in high-temperature and high-salt-concentration reservoirs.

\section{ACKNOWLEDGEMENTS}

I thank Professor GS Huang of the State Key Lab of Polymer Materials Engineering, Sichuan University, for her encouragement in commencing the research. The computation of the transition state was accomplished with assistance from $\mathrm{Dr} \mathrm{XY} \mathrm{Li}$ of the Center for Computation Chemistry and Biology, Sichuan University. I also thank Shengli Oilfield Sinopec for the financial assistance.

1 Ge, Z. \& Luo, Y. J. Synthesis, characterization, and properties of amphiphilic block copolymer of acrylamide-styrene by self-emulsifying microemulsion method. J. Appl. Polym. Sci. 114, 457-1463 (2009).

2 Beatriz, S. C., Dilon, L. P., Haag, R. B., Souza, C. R. \& Elizabete, F. L. Evaluation of hydrophobically associated polyacrylamide-containing aqueous fluids and their potential use in petroleum recovery. J. Appl. Polym. Sci. 91, 3686-3692 (2004).

$3 \mathrm{Ma}$, J. T., Huang, R. H., Zhao, L. \& Zhang, X. Solution properties of ionic hydrophobically associating polyacrylamide with an arylalkyl group. J. Appl. Polym. Sci. 97, 316-321 (2005)

4 Lu, H. S., Feng, Y. J. \& Huang, Z. Y. Association and effective hydrodynamic thickness of hydrophobically associating polyacrylamide through porous media. J. Appl. Polym. Sci. 110, 1837-1843 (2008).

5 Joseph, M., Tessymol, M., Devipriya, S. \& Kuriakose, S. Encapsulation and controlled release in polyacrylamide hydrogels. J. Appl. Polym. Sci. 93, 1816-1824 (2004).

6 Feng, Y. J., Grassl, B., Billon, L., Khoukh, A \& Francois, J Effects of $\mathrm{NaCl}$ on steady rheological behaviour in aqueous solutions of hydrophobically modified polyacrylamide and its partially hydrolyzed analogues prepared by post-modification. Polym. Int. 51, 939-947 (2002).

$7 \mathrm{Hu}$, S. W. \& Brittain, W. J. Surface grafting on polymer surface using physisorbed free radical initiator. Macromolecules. 38, 6592-6597 (2005).

8 Zhu, Z. Y., OY, J., Pallet, S., Desbrieres, J. \& Grassl, B. Hydrophobically modified associating polyacrylamide (HAPAM) synthesized by micellar copolymerization at higher monomer concentration. Euro. Polym. J. 43, 824-834 (2007).

9 Camail, M., Margaillan, A., Martin, I., Papailhou, A. L. \& Vernet, J. L. Synthesis of Nalkyl- and $\mathrm{N}$-arylalkylacrylamides and micellar copolymerization with acrylamide. Euro. Polym. J. 36, 1853-1863 (2000).

10 Pabon, M., Corpart, J. M, Selb, J \& Candau, F Synthesis in inverse emulsion and associating behavior of hydrophobically modified polyacrylamides. J. Appl. Polym. Sci. 91, 916-924 (2004).

$11 \mathrm{Li}$, H. Q. \& Ruckenstein, E. Amphiphilic particles with hydrophilic core/hydrophobic shell prepared via inverted emulsions. J. Appl. Polym. Sci. 61, 2129-2136 (1996).

12 Petit, L., Karakasyan, C., Pantoustier, N. \& Hourdet, D. Synthesis of graft polyacrylamide with responsive self-assembling properties in aqueous media. Polymer. 48, 7098-7112 (2007).

13 Zhang, Y. B., Wu, C., Fang, Q. \& Zhang, Y. X. A light-scattering study of the aggregation behavior of fluorocarbon-modified polyacrylamide in water. Macromolecules. 29, 2494-2497 (1996).

14 Tevenot, C., Bastaiat, G., Binana, W. \& Grassl, B. Aggregation number and critical micellar concentration of surfactant determined by time-dependent static light scattering and conductivity.. Coll. Surf. A. 25, 105-111 (2005).

15 Gao, B. J., Jiang, L. D. \& Liu, K. K. Microstructure and association property of hydrophobically modified polyacrylamide of a new family. Euro. Polym. J. 43, 45304540 (2007).

16 Chen, J., Du, L. B., Zhang, Y. X., Thieo, H. E. \& Jiang, M. Synthesis and solution properties of fluorocarbon-modified poly(N,N-dimethylacrylamide). Polym. Int. 50, 148-156 (2001).

17 Zhang, Y. X., Fang, Q., Fu, Y. Q., Da, A. H., Zhang, Y. B., Wu, C. \& Thieo, H. E. Synthesis and characterization of fluorocarbon-modified poly(N-isopropylacrylamide). Polym. Int. 49, 763-774 (2000).

18 Hoogerbrugge, P. J. \& Koelman, J.M.V.A. Simulation microscopic hydrodynamic phenomena with dissipative particle dynamics. Europhys. Lett. 19, 155-160 (1992).

19 Koelman, J.M.V.A. \& Hoogerbrugge, P.J. Dynamic simulation of hard-sphere suspension under steady shear. Europhys. Lett. 21, 363-369 (1993). 
20 Groot, R. D. Mesoscopic simulation of polymer-surfactant aggregation. Langmuir. 16, 7493-7502 (2000).

21 Yamamoto, S., Maruyama, Y. \& Hyodo, S. Dissipative particle dynamics study of spontaneous vesicle formation of amphiphilic molecules. J. Chem. Phys. 116, 5842 (2002).
22 Yan, L. T., Popp, N., Ghosh, S. K. \& Boker, A. Self-assembly of janus nanoparticles in diblock copolymers. ACS Nano. 4, 913-920 (2010).

$23 \mathrm{Li}$, X. J., Deng, M. G., Liu, Y. \& Liang, H. J. Dissipative particle dynamics simulations of toroidal structure formations of amphiphilic triblock copolymers. J. Phys. Chem. B. $112,14762-14765$ (2008).

Supplementary Information accompanies the paper on Polymer Journal website (http://www.nature.com/pj) 